\title{
Comparison between American Institute of Medicine Guidelines and Local Recommendation for Gestational Weight Gain in Taiwanese Primiparous Women
}

\author{
Alexander Waits \\ National Yang-Ming University \\ Chao-Yu Guo \\ National Yang-Ming University \\ Li-Yin Chien ( $\sim$ lychien@ym.edu.tw) \\ National Yang-Ming University https://orcid.org/0000-0002-4210-2975
}

\section{Research article}

Keywords: gestational weight gain, body mass index, Taiwan, maternal and child health, American Institute of Medicine

Posted Date: February 28th, 2020

DOl: https://doi.org/10.21203/rs.3.rs-15498/v1

License: (c) (1) This work is licensed under a Creative Commons Attribution 4.0 International License. Read Full License

Version of Record: A version of this preprint was published at Maternal and Child Health Journal on October 6th, 2021. See the published version at https://doi.org/10.1007/s10995-021-03231-2. 


\section{Abstract}

Background : American Institute of Medicine (IOM) recommends different ranges of gestational weight gain (GWG) based on pre-pregnancy body mass index (BMI). In Taiwan, IOM guidelines are implemented concurrently with the local recommendation for GWG (10-14 kg). This study compared between the two sets of guidelines in relation to adverse perinatal outcomes.

Methods : We analyzed 31653 primiparas with singletons from 2011-2016 annual National Breastfeeding Surveys. Logistic regressions for preterm birth, small for gestational age (SGA), large for gestational age (LGA), cesarean section and excessive postpartum weight retention (PWR) were fitted separately for GWG categorized according to IOM and Taiwan ranges. Areas under the receiver-operator curves (AUC) and the predicted probabilities for each outcome were compared in each BMI group.

Results : AUC for both guidelines ranged within $0.51-0.73$. Compared to Taiwan recommendation, IOM ranges showed lower probabilities of SGA for underweight ( $0.11-0.15$ versus $0.14-0.18)$, of LGA for obese $(0.12-0.15$ versus $0.15-0.18)$, of excessive PWR for overweight ( $0.19-0.30$ versus $0.27-0.39$ ), and obese (0.15- 0.22 versus $0.25-0.36)$; and higher probabilities of excessive PWR for underweight (0.170.33 versus $0.14-0.22$ )

Conclusions : Discriminative performance of IOM and Taiwan recommendations was poor for the five adverse birth outcomes, and no preference for either set of recommendations could be inferred from our results. In the absence of specific GWG guidelines, health care workers may provide inconsistent information to their patients. Future research is needed to explore optimal GWG ranges that can reliably predict locally relevant perinatal outcomes for mother and child.

\section{Introduction}

Many women and health providers are concerned with the question how much weight should be gained during pregnancy ${ }^{1}$. Gaining too much or not enough weight may affect maternal and child health during pregnancy, at delivery, and later in life, including increasing risks of obesity, cardiovascular diseases, and diabetes for mothers and their children ${ }^{2}$. The recommendations for GWG have changed dramatically over the past century ${ }^{1}$. The recent guidelines were developed by the American Institute of Medicine (IOM) in response to the obesity epidemic ${ }^{2}$. Based on the comprehensive literature review, optimal ranges for GWG were defined for each category of pre-pregnancy body mass index (BMI): $12.5-18 \mathrm{~kg}$ for $\mathrm{BMI}<18.5$; $11.5-16 \mathrm{~kg}$ for $18.5 \leq \mathrm{BMI}<25 ; 7-11.5 \mathrm{~kg}$ for $25 \leq \mathrm{BMI}<30$; and $5-9 \mathrm{~kg}$ for $\mathrm{BMI} \geq 30^{2}$. The women with GWG above the IOM recommendations had higher risks of cesarean section and excessive postpartum weight retention (PWR) (i.e., retaining more than $5 \mathrm{~kg}$ at 1 year postpartum). The infants born to those women could be at increased risk for presenting as large for gestational age (LGA), macrosomia, and childhood obesity. Women with GWG below the recommended ranges had higher risks of preterm birth, having a small for gestational age (SGA) baby, and failure to initiate breastfeeding ${ }^{2,3}$. Population-based 
survey revealed that only $32 \%$ of American mothers with full-term singletons were within the recommended range in 2010-2011 4 .

There is no global consensus about optimal GWG ranges. Recent review ${ }^{5}$ found that only 14 out of 70 developing and developed countries had some recommendations for GWG. Canada and Finland adopted IOM guidelines, while New Zealand adjusted IOM guidelines with different BMI cutoffs for its Caucasian $\left(18.5,25,30 \mathrm{~kg} / \mathrm{m}^{2}\right)$, Asian $\left(18.5,23,27.5 \mathrm{~kg} / \mathrm{m}^{2}\right)$ and Pacific $\left(18.5,26,32 \mathrm{~kg} / \mathrm{m}^{2}\right)$ populations.

Recommendations in other countries varied widely and were supported by limited evidence. Among the few countries in Asia with GWG recommendations, only Vietnam and Japan introduced BMI-specific ranges ${ }^{5}$.

Recommended GWG range in Taiwan was defined as $10-14 \mathrm{~kg}$, and IOM guidelines were added to the maternal health book, published by Taiwan Health Promotion Administration (HPA) in $2017^{6}$. Following World Health Organization (WHO) suggestion to develop local BMI cutoffs in Asian populations ${ }^{7}$, BMI cutoffs in Taiwan were set to 18.5, 24, and 27 to differentiate between underweight, normal, overweight, and obese ${ }^{8}$. So far, only few Taiwanese scholars examined GWG in relation to maternal and child health outcomes with controversial results, whereas some studies provided certain support for IOM guidelines 9-11, some found IOM guidelines inappropriate for Taiwanese women ${ }^{12}$. Despite the existence of

evidence-based BMI categorization for the local population ${ }^{13}$, Taiwanese studies mostly employed western BMI cutoffs, which may have resulted in misclassification bias. So far, none of the Taiwanese studies have used their data to compare between IOM and local GWG guidelines.

The objective of our study was to compare IOM and Taiwan GWG guidelines in a representative sample of Taiwanese women using receiver-operator curves and predicted probabilities for maternal and child adverse outcomes proposed by IOM in the latest revision of GWG guidelines: preterm birth, cesarean section, SGA, LGA, and excessive PWR ${ }^{2}$. Assessing discriminative performance of both guidelines is expected to provide an initial step in accumulating evidence for the development of local GWG guidelines in Taiwan.

\section{Methods}

\section{Study design and participants}

We used the 2011-2016 data from National Breastfeeding Surveys commissioned annually by Taiwan HPA. Detailed description of data collection was published earlier ${ }^{14}$. Structured questionnaires were delivered at 6-14 months postpartum to randomly selected mothers, who gave birth in those years. Primiparous mothers with singletons were included in the current study. Observations with missing data 
were excluded ( $n=3$ 471). After applying inclusion/exclusion criteria, we obtained the sample of 31653 primiparas with singletons, of whom $20.5 \%$ were underweight, $67.6 \%$ had normal pre-pregnancy BMI, $8.3 \%$ were overweight and $4.8 \%$ were obese (Figure 1 ).

\section{Measurements}

The dependent variables in our study included preterm birth, SGA, LGA, cesarean section, and excessive PWR based on the IOM review: ${ }^{2}$. Preterm birth was defined as delivery at less than 37 gestational weeks. SGA and LGA were defined as infant birth weight less than the $10^{\text {th }}$ percentile or more than the $90^{\text {th }}$ percentile, respectively, adjusted for gestational week and infant sex. The percentiles were obtained from Taiwan national intrauterine growth charts for live-born singletons delivered between 22 and 42 weeks ${ }^{15}$. Cesarean section was confirmed through the survey questions: 1) was the delivery natural or cesarean? 2) was there a medical indication for cesarean section? Since non-medical indications accounted for less than $2 \%$ of all cesarean sections, and sensitivity analysis without these cases did not affect our results, we included all cesarean sections in the final analysis. Excessive PWR was calculated by subtracting prepregnancy weight from the current weight reported in the interview and was defined as $\geq 5 \mathrm{~kg}^{2}$. Although women in our sample were interviewed at 6 to 14 months postpartum, there was no significant difference in the proportion of excessive PWR among the postpartum months $\left(\chi^{2}\right.$ test $\left.p=0.354\right)$.

Pre-pregnancy BMI was calculated from self-reported weight and height and categorized with Taiwanese cutoffs as underweight (BMl<18.5), normal (18.5 $\leq \mathrm{BMI}<24)$, overweight $(24 \leq \mathrm{BMI}<27)$, and obese $(\mathrm{BMI} \geq 27)^{8}$. Inadequate, appropriate, and excessive GWG was categorized according to IOM guidelines (12.5-18 kg for $B M l<18.5,11.5-16 \mathrm{~kg}$ for $18.5 \leq \mathrm{BMl}<25,7-11.5 \mathrm{~kg}$ for $25 \leq \mathrm{BMl}<30,5-9 \mathrm{~kg}$ for $\mathrm{BMI} \geq 30)^{2}$, and according to Taiwan HPA recommendation of $10-14 \mathrm{~kg}$ regardless of pre-pregnancy BMI ${ }^{6}$.

We selected maternal age, education, country of origin, employment, infant sex, year of birth, and health status at birth as potential confounding variables in statistical models, based on literature review ${ }^{16-18}$ and significant associations between these variables and our dependent variables.

\section{Statistical analysis}

Mean and standard deviation of GWG were calculated for each BMI group, and analysis of variance with least significant difference between pair-wise means was adjusted using Bonferroni's correction for multiple testing. All other variables were cross-tabulated by BMI status, and $\chi^{2}$ tests were performed to compare the proportions among the BMl groups.

Two logistic regression models were fit for each of the five outcomes (preterm delivery, cesarean section, SGA, LGA, and excessive PWR). The independent variable of GWG was categorized according to the IOM in one model and according to the Taiwan GWG recommendation (10-14 kg) in the other model. Both models were adjusted for maternal age, education, country of origin, employment, infant sex, health status at birth, and year of birth. We estimated discriminative performance of IOM and Taiwan GWG 
categorizations with receiver-operator curves for each logistic regression by calculating area under the curve (AUC). Discrimination was considered poor if AUC estimate was between 0.5 and 0.7 and acceptable if AUC was 0.7 or higher ${ }^{19}$. Comparison between AUC for IOM and Taiwan GWG categorizations was based on $p$-values for Wald statistics ${ }^{20}$. The above statistical procedure was performed for the total sample size and for each BMI group. Odds ratios and $95 \%$ confidence intervals (Cl) are presented in the supplementary materials (Supplementary Table S1).

We also fitted adjusted logistic regression models for each BMI group with GWG as a continuous variable in order to calculate the predicted probabilities with $95 \% \mathrm{Cl}$ for each of the five outcomes (Supplementary Tables S2-S6). Predicted probabilities and 95\% Cl were plotted against GWG within the range of 4-20 kg. Overlapping $\mathrm{Cl}$ suggested non-significant differences between IOM and Taiwan GWG ranges.

Level of significance was set to a two-sided $a=0.05$ in all statistical procedures. The missing values comprised $9.9 \%$ of the analytical sample. A sensitivity analysis, with 100 multiple imputations by chained equations ${ }^{21}$, did not alter our findings and conclusions; therefore, we present initial results after casewise deletion. All the analyses were performed with Stata Statistical Software: Release 15 (Stata Corp. 2017. College Station, TX: Stata Corp LLC).

\section{Results}

Mean GWG did not differ significantly between the underweight (14.1 \pm 5.0$)$ and normal BMI groups (14.2 \pm 5.0$)$; however, it was significantly lower in the overweight $(13.1 \pm 5.8)$ and the obese $(11.2 \pm 6.4 \mathrm{~kg})$ groups. The underweight group showed the highest proportion of SGA (15.2\% versus $8.8-10.8 \%)$, while the obese group showed the highest proportion of preterm birth (9.7\% versus $5.9-7.1 \%)$, LGA $(17.1 \%$ versus $4.3-12.4 \%)$, and caesarean section (50.2\% versus $28.3-40.5 \%)$. The highest percentage of excessive PWR (37.5\% versus 24.4-30.6\%) was observed in the overweight group. Underweight group tended to be younger than 29, while obese group tended to be 35 or older, and the age group of 30-34 had the highest percentage of women with normal BMI (44.9\% versus 39.0-42.1\%). Pre-pregnancy BMI also differed significantly $(p<0.001)$ by education, employment, country of origin, and newborn health status at birth (Table 1).

GWG status differed significantly $\left(\chi^{2}\right.$ test $p<0.001$ ) between BMI groups within both IOM and Taiwan categorizations. Percentages of appropriate GWG according to IOM ranged between $33.3 \%$ in the obese and $40.5 \%$ in the underweight, which were similar to the values obtained based on Taiwan GWG categorization: $33.6 \%$ in the obese and $41.7 \%$ in the underweight. IOM categorization showed higher percentages of inadequate GWG in the underweight (41.9\%) and excessive GWG in the obese $(50.2 \%)$ compared to the percentages obtained from Taiwan categorization (15.8\% and $27.8 \%$, respectively). Within the normal BMI group, a greater proportion of mothers were categorized as having excessive GWG according to the Taiwan recommendation (44.2\% versus $29.0 \%$ ), while the IOM categorization revealed a higher percentage (30.9\% versus $15.3 \%$ ) of inadequate GWG (Figure 2 ). 


\section{Preterm birth}

Both GWG categorizations showed AUC $<0.70$ for preterm birth. Taiwan categorization showed significantly $(p<0.001)$ higher AUC in the total study sample $(0.64$ versus 0.63$)$ and in the underweight group (0.61 versus 0.57 ) (Table 2).

Predicted probability of preterm birth decreased with each additional kilogram of GWG, from 0.10 at $4 \mathrm{~kg}$ to 0.04 at $20 \mathrm{~kg}$, for the normal BMI group. No significant differences were observed among the underweight, normal, and overweight groups. Within the obese group, those who gained more than $13 \mathrm{~kg}$ had a significantly higher probability (0.09) of preterm birth than the other BMI groups (Figure 3A).

IOM and Taiwan GWG ranges showed overlapping probabilities of preterm birth in the normal, overweight, and obese groups. A slightly significant variation between the IOM and Taiwan ranges was observed in the underweight $\mathrm{BMI}$ group, with preterm birth probabilities ranging from 0.06 (95\% $\mathrm{Cl}: 0.06-0.07)$ to 0.04 (95\% Cl: $0.03-0.04)$ within the IOM range $(12.5-18 \mathrm{~kg})$ and from 0.08 (95\% Cl: $0.07-0.08)$ to 0.05 (95\% Cl: $0.05-0.06)$ within the Taiwan range (10-14 kg) (Figure 3A, Supplementary Table S2).

\section{Small for gestational age}

Both GWG categorizations showed AUC $<0.60$ for SGA. IOM categorization showed significantly $(p<0.010)$ higher AUC in the total study sample (0.59 versus 0.58 ) (Table 2 ).

Predicted probability of SGA decreased with each additional kilogram of GWG from 0.17 at $4 \mathrm{~kg}$ to 0.08 at $20 \mathrm{~kg}$ for normal BMI. No significant differences were observed between the overweight and obese groups. The group with normal BMI displayed significantly higher probabilities of SGA $(\geq 0.12)$ than the overweight $(\geq 0.10)$ and obese groups $(\geq 0.08)$ if less than $12 \mathrm{~kg}$ were gained during gestation. Underweight, who gained less than $18 \mathrm{~kg}$ had significantly higher probability of SGA $(\geq 0.11)$ than the normal ( $\geq 0.09)$, overweight $(\geq 0.08)$ and obese $(\geq 0.07)$ groups (Figure $3 B)$.

Probabilities of SGA overlapped for IOM and Taiwan GWG ranges in the normal, overweight and obese groups. A significant variation between IOM and Taiwan ranges was observed in the underweight BMI group, with SGA probabilities ranging from 0.15 (95\% Cl: $0.14-0.16)$ to 0.11 (95\% Cl: $0.10-0.12)$ within the IOM range (12.5-18 kg) and from $0.18(95 \% \mathrm{Cl}: 0.17-0.20)$ to $0.14(95 \% \mathrm{Cl}: 0.13-0.15)$ within the Taiwan range (10-14 kg) (Figure 3B, Supplementary Table S3).

\section{Large for gestational age}

Both GWG categorizations showed AUC $<0.70$ for LGA. IOM categorization showed significantly higher AUC in the total study sample $(0.65$ versus $0.62, p<0.001)$ and in the normal BMI group $(0.60$ versus 0.59 , $\mathrm{p}=0.008)($ Table 2).

Predicted probability of LGA increased with each additional kilogram of GWG, from 0.03 at $4 \mathrm{~kg}$ to 0.10 at $20 \mathrm{~kg}$, for the normal BMI group. The underweight group had significantly lower probability $(0.01-0.07)$ 
within the range of $4-20 \mathrm{~kg}$, while the overweight (0.08-0.15) and obese (0.12-0.24) groups had significantly higher probabilities than the normal BMI groups (0.03-0.10) (Figure 3C).

Probabilities of LGA overlapped for IOM and Taiwan GWG ranges in the underweight, normal, and overweight groups. A slightly significant variation between the IOM and Taiwan ranges was observed in the obese BMI group, with LGA probabilities ranging from 0.12 (95\% Cl: $0.10-0.15)$ to 0.15 (95\% Cl: $0.13-$ $0.17)$ within the IOM range $(5-9 \mathrm{~kg})$ and from 0.15 (95\% Cl: $0.13-0.17)$ to 0.18 (95\% Cl: $0.16-0.21)$ within the Taiwan range (10-14 kg) (Figure 3C, Supplementary Table S4).

\section{Cesarean section}

IOM categorization showed significantly $(\mathrm{p}<0.010)$ higher AUC for cesarean section in the total study sample (0.73 versus 0.72 ), whereas AUC estimates in BMI groups did not exceed 0.55 and did not differ between IOM and Taiwan GWG categorizations (Table 2).

Predicted probability of cesarean section increased with each additional kilogram of GWG, from 0.26 at 4 $\mathrm{kg}$ to 0.36 at $20 \mathrm{~kg}$, for the normal BMI group. The underweight group had significantly lower probability (0.22-0.32) of cesarean section within the range of $4-20 \mathrm{~kg}$, while the overweight group (0.35-0.47) and obese (0.45-0.54) group had significantly higher probabilities of cesarean section than the normal BMI group (0.26-0.36) (Figure 3D).

Probabilities of cesarean section overlapped for IOM and Taiwan GWG ranges in the underweight, normal, and overweight groups. A slightly significant variation between the IOM and the Taiwan ranges was observed in the obese group, with probabilities for cesarean section ranging from 0.46 ( $95 \% \mathrm{Cl}$ : $0.42-$ $0.59)$ to 0.48 (95\% Cl: $0.45-0.51)$ within the IOM range (5-9 kg), and from 0.49 (95\% Cl: $0.46-0.51)$ to 0.51 (95\% Cl: 0.48-0.54) within the Taiwan range (10-14 kg) (Figure 3D, Supplementary Table S5).

\section{Excessive postpartum weight retention}

IOM categorization showed significantly higher AUC for excessive PWR in the total study sample $(0.69$ versus $0.68, p<0.001)$ and in the normal BMI group (0.67 versus $0.66, p<0.001)$. AUC for Taiwan categorization were significantly higher in the overweight $(0.69$ vs $0.67, p 0.026)$ and in obese $(0.70$ vs $0.67, \mathrm{p}=0.001$ ) groups (Table 2).

Predicted probability of excessive PWR increased with each additional kilogram of GWG, from 0.08 at 4 $\mathrm{kg}$ to 0.49 at $20 \mathrm{~kg}$, for the normal BMl group. The underweight group had significantly lower probability $(\geq 0.09)$ than the normal BMl group, if more than $7 \mathrm{~kg}$ were gained during gestation. The overweight (0.13-0.61) and obese (0.13-0.55) groups displayed significantly higher probabilities than the normal BMI group (0.08-0.49); however, there was no significant difference between the overweight and obese groups (Figure 3E).

Significant differences between the predicted probabilities for excessive PWR between IOM and Taiwan ranges were observed in all BMI groups. The probabilities in the underweight group ranged from 0.17 
(95\% Cl: $0.16-0.19)$ to 0.33 (95\% Cl: $0.31-0.34)$ within the IOM range $(12.5-18 \mathrm{~kg})$ and from 0.14 (95\% Cl: $0.12-0.15)$ to 0.22 ( $95 \% \mathrm{Cl}: 0.21-0.23)$ within the Taiwan range $(10-14 \mathrm{~kg})$. The probabilities in the normal BMI group ranged from 0.20 ( $95 \% \mathrm{Cl}: 0.19-0.20)$ to 0.34 (95\% Cl: $0.33-0.35)$ within the IOM range (11.5-16 kg) and from 0.17 (95\% Cl: $0.17-0.18)$ to 0.28 (95\% Cl 0.27-0.28) within the Taiwan range (10-14 kg). The probabilities in the overweight BMI group ranged from 0.19 (95\% Cl: $0.16-0.21)$ to 0.30 (95\% Cl: $0.27-0.32)$ within the IOM range (7-11.5 kg) and from 0.27 (95\% Cl: $0.24-0.29)$ to 0.39 (95\% Cl: $0.27-0.42)$ within the Taiwan range $(10-14 \mathrm{~kg})$. The probabilities in the obese group ranged from 0.15 (95\% Cl: $0.12-0.17)$ to 0.22 (95\% Cl: $0.20-0.25)$ within the IOM range (5-9 kg), and from 0.25 (95\% Cl: $0.22-0.27)$ to $0.36(95 \% \mathrm{Cl}: 0.33-0.39)$ within the Taiwan range (10-14 kg) (Figure 3E, Supplementary Table S6).

\section{Discussion}

To date, this study is the first to compare the Taiwan and the IOM guidelines for GWG in a large national sample of primiparas with singletons. The recommended range of GWG in Taiwan categorized more obese as gaining inadequate weight (38.6\%) and more underweight as gaining excessive weight (42.5\%), while IOM ranges categorized more underweight as gaining inadequate weight $(41.9 \%)$ and more obese as gaining excessive weight (50.2\%) (Figure 2). This discrepancy in GWG categorization is likely due to the fact that the IOM ranges are BMI-specific, which were established to address the obesity epidemic in the United States ${ }^{2}$. While obesity is a global issue, the BMI distribution in Asian countries is different compared to the BMI distribution in the West ${ }^{7}$. Along with the increasing prevalence of women entering pregnancy with an excessive $\mathrm{BMI}$, there is also a high proportion of women who are underweight at the beginning of gestation, as we observed in our previous study based on a nationally representative sample of Taiwanese women (under review, data not shown). High prevalence of underweight women that do not gain an adequate amount of weight during pregnancy has been reported in Taiwan, Korea, and Japan $16,17,22,23$. The BMI-specific ranges of GWG recommended by IOM appear to tackle this issue more effectively, compared to the GWG range recommended by Taiwan HPA, by categorizing a greater proportion of underweight women as having inadequate GWG.

Although there were significant, albeit small, differences in AUC between IOM and Taiwan categorizations in the total study sample, often there were no significant differences between both GWG categorizations within the BMI groups. Taiwan guidelines were slightly better at discriminating the prevalence of preterm birth in the underweight group (AUC 0.61 versus 0.57 ), and excessive PWR in the overweight (AUC 0.69 versus 0.67 ) and obese (AUC 0.70 versus 0.67 ) groups. Nevertheless, the discriminative performance of both classification systems was rather poor, as indicated by AUC $\leq 0.70$ for the four adverse outcomes, and only $0.72-0.73$ for cesarean section in the total study sample (Table 2). Previously, a Japanese study reported AUC of 0.61 and 0.62 for SGA and LGA, respectively ${ }^{17}$. Another study in Latin-American women similarly showed poor discriminative performance $(A U C<0.70)$ of weekly BMI gain during pregnancy for low birth weight, SGA and LGA ${ }^{24}$. Most recent and comprehensive meta-analysis of 25 cohort studies also showed low ability of IOM categorization to distinguish between women with and 
without perinatal adverse outcomes, with AUC ranging from 0.55 to 0.60 for preterm birth, SGA, LGA, and cesarean section ${ }^{25}$. The multifactorial nature of the adverse birth outcomes, as well as unmeasured confounding, could contribute to low estimates of AUC. Another possible reason for low AUC estimates may stem from the fact that our study population included respondents to breastfeeding surveys. Since adverse birth outcomes could hamper breastfeeding ${ }^{26}$, mothers who did not breastfeed may have experienced some of the birth outcomes of interest in our study and chose not to participate. However, previous studies that assessed the general population of pregnancies unrelated to breastfeeding yielded similar results $17,24,25$.

Based on the predictive probabilities of the adverse birth outcomes, the recommended GWG range of 10$14 \mathrm{~kg}$ in Taiwan was more protective for overweight and obese women regarding preterm birth and SGA, as well as for women in the underweight and normal BMI groups, specifically LGA, cesarean section, and excessive PWR. IOM ranges were more protective for preterm birth and SGA in the underweight and normal BMI groups; and for LGA, cesarean section, and excessive PWR in the overweight and obese groups (Figure 3). To date, no previous study in Taiwan has performed a direct comparison of IOM and Taiwan GWG guidelines; however, risk assessment studies yielded results that are consistent with our findings. A large retrospective cohort study between 2009 and 2015 in a hospital that applied IOM guidelines and the western BMI cutoffs reported that GWG below the IOM guidelines increased the risk of SGA for women in the underweight $(\mathrm{BMl}<18.5)$ or normal weight $(18.5 \leq \mathrm{BMl}<25)$ groups, but not for women in the overweight $(25 \leq \mathrm{BMl}<30)$ and obese $(\mathrm{BMl} \geq 30)$ groups ${ }^{10}$. Another study that applied the Taiwan classification of GWG and BMI in case-control studies reported that normal $(18.5 \leq \mathrm{BML}<25)$ and underweight $(B M \mid<18.5)$ women who adhere to the IOM guidelines had a reduced risk of infant low birth weight ${ }^{11}$; however, following IOM guidelines for normal $(18.5 \leq \mathrm{BMK}<25)$ and overweight $(25 \leq \mathrm{BMK}<30)$ women could lead to an increased risk of macrosomia ${ }^{12}$.

Our study assessed five adverse birth outcomes, which were selected by the IOM committee for their relevance in the American population, and for the well-established associations with GWG ${ }^{2}$. Without undermining the importance of those adverse birth outcomes for maternal and child health, the focus of public health may shift in Asian populations. While the incidence of low birth weight, preterm birth, and SGA is increasing, macrosomia (about 1\%) and LGA are less prevalent in Taiwan ${ }^{27}$. Additional birth outcomes were associated with GWG in the Taiwanese population. Pre-pregnancy BMI of 18.5-24 followed by GWG of 10-14 kg could lead to better obstetric management, including reduced risks of low birth weight, preterm labor, cesarean delivery, preeclampsia, and gestational diabetes mellitus ${ }^{28}$. In a cohort of 1147 women from Taipei, maternal (gestational diabetes mellitus, dysfunctional labor, and cesarean delivery) and neonatal complications (macrosomia and meconium aspiration) were significantly associated with excessive GWG, but not with inadequate GWG, based on the IOM classification ${ }^{9}$. In addition, GWG below the values stipulated in the IOM guidelines was an independent risk factor for placental abruption, while preeclampsia was related to pre-pregnancy obesity, but not to excessive GWG ${ }^{10}$. A way to account for the trade-off between the outcomes could involve weighting technique according to the importance of the outcomes as agreed by specialists, e.g. obstetricians, 
neonatologists, NICU nurses, etc. For example, SGA was selected as the least adverse outcome, while preterm birth, complicated delivery and preeclampsia were assigned 1.2-2.3 times higher weights by Japanese specialists ${ }^{16}$.

Previous studies in Western and Asian populations have shown that pre-pregnancy weight status and GWG could be independent predictors of birth outcomes for mothers and infants $16,18,25,29,30$. In the absence of clear guidelines in Taiwan, pre-pregnancy BMI could be a more relevant target for public health interventions. Our results indicated that the normal BMI group had substantially lower probabilities of all the five outcomes than the overweight and obese groups. Therefore, entering pregnancy at normal BMI, with local cutoffs of $18.5-24 \mathrm{~kg} / \mathrm{m}^{2}$, should be advised.

Our study analyzed a large national sample, ensuring high generalizability of our results for the Taiwanese population and adequate statistical power of our analyses. However, our study presents the following limitations. First, our study population was likely to be healthier than the general population, due to the possibility of the non-participation of mothers with adverse birth outcomes. Second, using BMI for classification of weight status could lead to misclassification bias. BMI was reported to be a limited indicator of obesity and body composition, as people with lower BMI could still have levels of adiposity equivalent to those found in obese individuals when measured by bioelectrical impedance ${ }^{31}$. Although we applied local, empirically established cutoffs for BMI classification to reduce the risk of misclassification, the use of waist circumference, or bioelectrical impedance, for more accurate results is recommended in the future. Self-reported height and weight also could bias BMI estimates, although prepregnancy BMI based on self-reported values was shown as a reliable and valid estimate for populationbased research and surveillance purposes ${ }^{32}$. Residual confounding was likely due to the absence of information regarding every potential confounder, including smoking and environmental exposures ${ }^{33}$. Taiwan Birth Cohort Study detected only 3\% of smokers in a sample of Taiwanese pregnant women in $2005^{34}$. Assuming that our sample represents a healthier population, the prevalence of smokers would likely to be even lower, and therefore, the effect of smoking would be undetectable.

\section{Conclusions}

We observed poor discriminative performance of the IOM and the Taiwan guidelines for GWG regarding the five adverse birth outcomes for mother and infant. Also, a comparison between the predicted probabilities for each outcome did not support a preference for either set of recommendations. Physicians and health care workers need to individualize prenatal advice regarding GWG while following up-to-date research in local and world populations. Future research in large representative samples is required to assess optimal GWG ranges for Asian women and adverse perinatal outcomes, which are relevant to the local population.

\section{Abbreivations}

AUC - area under the receiver-operator curve 
$\mathrm{BMI}$ - body mass index

GWG - gestational weight gain

HPA - Taiwan health promotion administration

IOM - American institute of medicine

LGA - large for gestational weight

PWR - postpartum weight retention

SGA - small for gestational weight

\section{Declarations}

Ethics approval and consent to participate: The study was based on the secondary data. Original surveys were ethically approved by the joint institutional review board. Our study used anonymous data without linkable ID.

Consent for publication: All authors give their consent for publication

Competing interests: No competing interests to report

Funding: No funding to report

Authors' contributions: CLY conceptualized the study and reviewed the manuscript, CGY performed statistical analysis and reviewed the manuscript, AW performed statistical analysis and wrote the manuscript

Acknowledgements: The data for this study were obtained from Taiwan National Breastfeeding Surveys, commissioned by the Health Promotion Administration, Ministry of Health and Welfare, Taiwan. We would like to thank the Health Promotion Administration, Ministry of Health and Welfare, Taiwan for providing the data for our study.

\section{References}

1. Kominiarek MA, Peaceman AM. Gestational weight gain. American journal of obstetrics and gynecology. 2017.

2. Yaktine AL, Rasmussen KM, eds. Weight Gain During Pregnancy: Reexamining the Guidelines. Washington, D.C.: National Academies Press; 2009.

3. Siega-Riz AM, Viswanathan M, Moos M-K, et al. A systematic review of outcomes of maternal weight gain according to the Institute of Medicine recommendations: birthweight, fetal growth, and 
postpartum weight retention. American journal of obstetrics and gynecology. 2009;201(4):339. e331339. e314.

4. Deputy NP, Sharma AJ, Kim SY, Hinkle SN. Prevalence and characteristics associated with gestational weight gain adequacy. Obstetrics and gynecology. 2015;125(4):773.

5. Alavi N, Haley S, Chow K, McDonald S. Comparison of national gestational weight gain guidelines and energy intake recommendations. Obesity Reviews. 2013;14(1):68-85.

6. Taiwan Health Promotion Administration. Maternal Health booklet. In: Taiwan Ministry of Health and Welfare, ed. Taipei2017.

7. Barba C, Cavalli-Sforza T, Cutter J, Darnton-Hill I. Appropriate body-mass index for Asian populations and its implications for policy and intervention strategies. The lancet. 2004;363(9403):157.

8. Taiwan Health Promotion Administration. BMI classification (in Chinese). http://health99.hpa.gov.tw/OnlinkHealth/Onlink_BMl.aspx. Published 2018. Accessed.

9. Horng H-C, Huang B-S, Lu Y-F, et al. Avoiding excessive pregnancy weight gain to obtain better pregnancy outcomes in Taiwan. Medicine. 2018;97(4).

10. Hung TH, Hsieh TT. Pregestational body mass index, gestational weight gain, and risks for adverse pregnancy outcomes among Taiwanese women: A retrospective cohort study. Taiwanese journal of obstetrics \& gynecology. 2016;55(4):575-581.

11. Tsai YL, Chen LC, Seow KM, Chong KM. The recommendations of the American Institute of Medicine (IOM) for normal and underweight women to reduce the risk of low birth weight. Taiwanese journal of obstetrics \& gynecology. 2015;54(1):1-7.

12. Tsai YL, Chong KM, Seow KM. Following the 2009 American Institute of Medicine recommendations for normal body mass index and overweight women led to an increased risk of fetal macrosomia among Taiwanese women. Taiwanese journal of obstetrics \& gynecology. 2013;52(3):341-346.

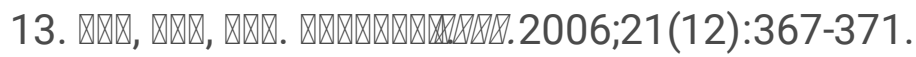

14. Waits A, Guo CY, Chien LY. Evaluation of factors contributing to the decline in exclusive breastfeeding at 6 months postpartum: The 2011-2016 National Surveys in Taiwan. Birth (Berkeley, Calif). 2018;45(2):184-192.

15. Hsieh WS, Wu HC, Jeng SF, et al. Nationwide singleton birth weight percentiles by gestational age in Taiwan, 1998-2002. Acta paediatrica Taiwanica = Taiwan er ke yi xue hui za zhi. 2006;47(1):25-33.

16. Morisaki N, Nagata C, Jwa SC, et al. Pre-pregnancy BMI-specific optimal gestational weight gain for women in Japan. Journal of Epidemiology. 2017.

17. Nomura K, Kido M, Tanabe A, Nagashima K, Takenoshita S, Ando K. Investigation of optimal weight gain during pregnancy for Japanese Women. Scientific Reports. 2017;7.

18. Ota $E$, Haruna $M$, Suzuki M, et al. Maternal body mass index and gestational weight gain and their association with perinatal outcomes in Viet Nam. Bulletin of the World Health Organization. 2011;89(2):127-136.

19. Hosmer DW, Lemeshow S, Sturdivant RX. Applied Logistic Regression. Wiley; 2013. 
20. Pepe M, Longton G, Janes H. Estimation and Comparison of Receiver Operating Characteristic Curves. The Stata journal. 2009;9(1):1-1.

21. Sterne JAC, White IR, Carlin JB, et al. Multiple imputation for missing data in epidemiological and clinical research: potential and pitfalls. The BMJ. 2009;338:b2393.

22. ha Wie J, Park IY, Namkung J, Seo HW, Jeong MJ, Kwon JY. Is it appropriate for Korean women to adopt the 2009 Institute of Medicine recommendations for gestational weight gain? PloS one. 2017;12(7):e0181164.

23. Hung TH, Chen SF, Hsu JJ, Hsieh TT. Gestational weight gain and risks for adverse perinatal outcomes: A retrospective cohort study based on the 2009 Institute of Medicine guidelines. Taiwanese journal of obstetrics \& gynecology. 2015;54(4):421-425.

24. Kac G, Nucci LB, Spyrides MHC, Duncan BB, Schmidt MI. Evaluation of the ability of a LatinAmerican gestational weight curve to predict adverse pregnancy outcomes. International Journal of Gynecology \& Obstetrics. 2009;106(3):223-226.

25. Voerman E, Santos S, Inskip H, et al. Association of Gestational Weight Gain With Adverse Maternal and Infant Outcomes. Jama. 2019;321(17):1702-1715.

26. Sharma IK, Byrne A. Early initiation of breastfeeding: a systematic literature review of factors and barriers in South Asia. International breastfeeding journal. 2016;11:17.

27. Health Promotion Administration T. 2016 Statistics of Birth Reporting System. In:2017.

28. Tsai IH, Chen CP, Sun FJ, Wu CH, Yeh SL. Associations of the pre-pregnancy body mass index and gestational weight gain with pregnancy outcomes in Taiwanese women. Asia Pacific journal of clinical nutrition. 2012;21(1):82-87.

29. Nohr EA, Vaeth M, Baker JL, Sørensen TI, Olsen J, Rasmussen KM. Pregnancy outcomes related to gestational weight gain in women defined by their body mass index, parity, height, and smoking status. The American journal of clinical nutrition. 2009;90(5):1288-1294.

30. Oken E, Kleinman KP, Belfort MB, Hammitt JK, Gillman MW. Associations of gestational weight gain with short-and longer-term maternal and child health outcomes. American journal of epidemiology. 2009;170(2):173-180.

31. Frankenfield DC, Rowe WA, Cooney RN, Smith JS, Becker D. Limits of body mass index to detect obesity and predict body composition. Nutrition. 2001;17(1):26-30.

32. Shin D, Chung H, Weatherspoon L, Song WO. Validity of prepregnancy weight status estimated from self-reported height and weight. Maternal and child health journal. 2014;18(7):1667-1674.

33. Kramer MS. Determinants of low birth weight: methodological assessment and meta-analysis. Bull World Health Organ. 1987;65(5):663-737.

34. Ko TJ, Tsai LY, Chu LC, et al. Parental smoking during pregnancy and its association with low birth weight, small for gestational age, and preterm birth offspring: a birth cohort study. Pediatrics and neonatology. 2014;55(1):20-27. 


\section{Tables}

Table 1. Characteristics of Taiwanese primiparous women with singleton pregnancies based on pre-pregnancy body mass index $(\mathrm{N}=31653)$.

ANOVA - analysis of variance, BMI - body mass index, SD - standard deviation

Table 2. Comparison of areas under receiver-operator curves for the American Institute of Medicine guidelines and the Taiwan recommendation for gestational weight gain $(\mathrm{N}=31653)$.

AUC - area under the curve, BMI - pre-pregnancy body mass index, IOM - American Institute of Medicine, LGA - large for gestational age, PWR - postpartum weight retention SGA - small for gestational age

Figures

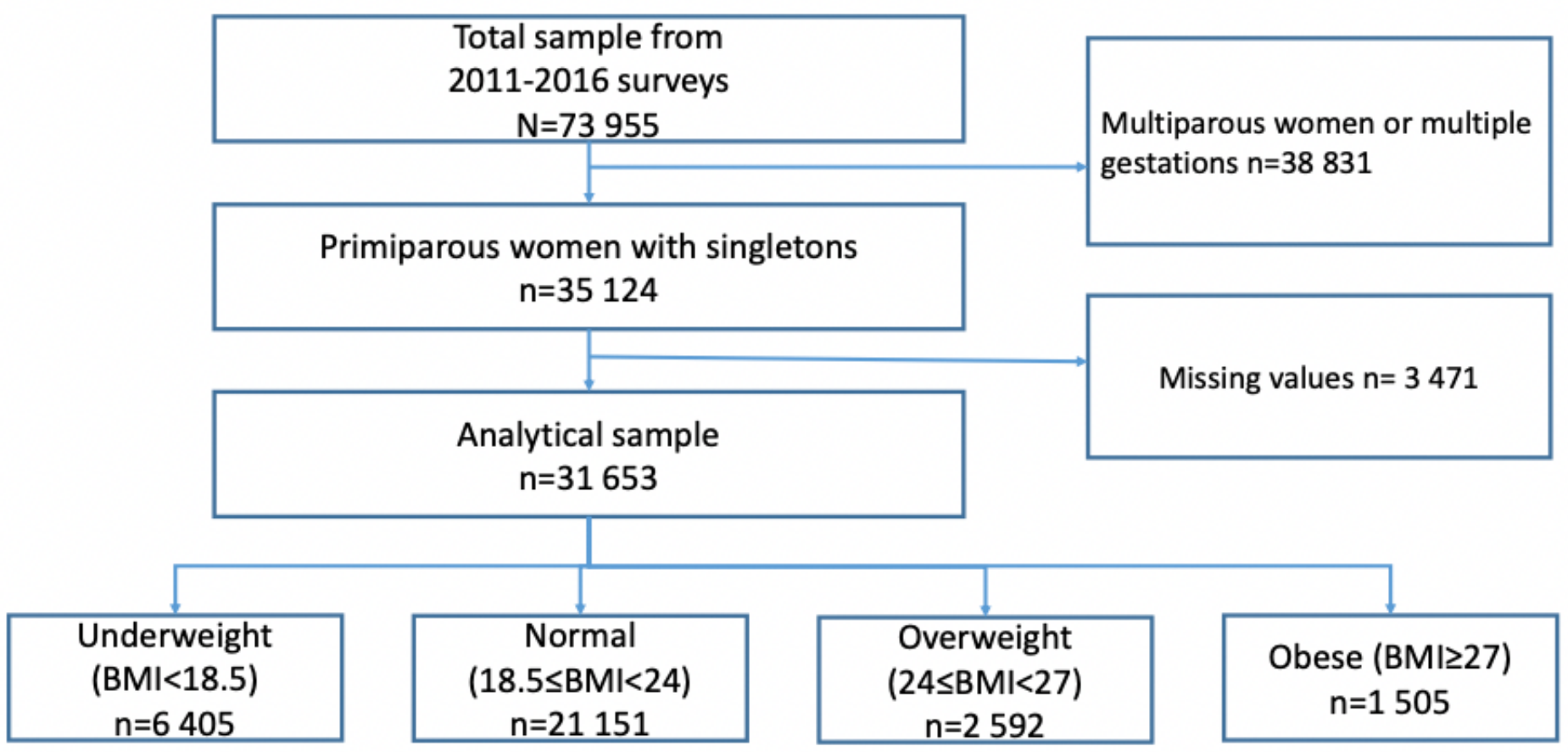

Figure 1

Flowchart of sample selection 


\begin{tabular}{|c|c|c|c|c|c|c|}
\hline & All & $\begin{array}{l}\text { Underweight } \\
\mathrm{BMI}<18.5\end{array}$ & $\begin{array}{c}\text { Normal } \\
18.5 \leq \mathrm{BMI}<24\end{array}$ & $\begin{array}{l}\text { Overweight } \\
24 \leq \mathrm{BMI}<27\end{array}$ & $\begin{array}{c}\text { Obese } \\
\mathrm{BMI} \geq 27\end{array}$ & $\begin{array}{c}\mathrm{p} \text { - } \\
\text { values }\end{array}$ \\
\hline & $\mathrm{N}=31,653$ & $\mathrm{n}=6,405$ & $\mathrm{n}=21,151$ & $\mathrm{n}=2,592$ & $\mathrm{n}=1,505$ & \multirow[t]{2}{*}{ ANOVA } \\
\hline & mean $\pm S D$ & mean $\pm S D$ & mean $\pm S D$ & mean $\pm S D$ & mean $\pm S D$ & \\
\hline Gestational weight gain, kg & $13.9 \pm 5.2$ & $14.1 \pm 5.0$ & $14.2 \pm 5.0)$ & $13.1 \pm 5.8$ & $11.2 \pm 6.4$ & $<0.001$ \\
\hline & $\%(n)$ & $\%(n)$ & $\%(n)$ & $\%(n)$ & $\%(n)$ & $\chi^{2}$ test \\
\hline Preterm delivery & $6.2(1,965)$ & $6.0(382)$ & $5.9(1,254)$ & $7.1(183)$ & $9.7(146)$ & $<0.001$ \\
\hline Small for gestational age & $11.5(3,653)$ & $15.2(972)$ & $10.8(2,285)$ & $10.2(264)$ & $8.8(132)$ & $<0.001$ \\
\hline Large for gestational age & $7.7(2,423)$ & $4.3(277)$ & $7.4(1,568)$ & $12.4(321)$ & $17.1(257)$ & $<0.001$ \\
\hline Cesarean section & $33.0(10,454)$ & $28.3(1,811)$ & $32.3(6,839)$ & $40.5(1,049)$ & $50.2(755)$ & $<0.001$ \\
\hline $\begin{array}{l}\text { Excessive postpartum weight } \\
\text { retention }\end{array}$ & $29.8(9,446)$ & $24.4(1,562)$ & $30.5(6,452)$ & $37.5(972)$ & $30.6(460)$ & $<0.001$ \\
\hline Age & & & & & & $<0.001$ \\
\hline $20-24$ & $8.5(2,702)$ & $13.9(890)$ & $7.2(1,529)$ & $6.8(175)$ & $7.2(108)$ & \\
\hline $25-29$ & $30.0(9,480)$ & $35.8(2,290)$ & $28.7(6,064)$ & $27.7(717)$ & $27.2(409)$ & \\
\hline $30-34$ & $43.3(13,697)$ & $39.0(2,499)$ & $44.9(9,501)$ & $42.1(1,091)$ & $40.3(606)$ & \\
\hline 35 or older & $18.2(5,774)$ & $11.3(726)$ & $19.2(4,057)$ & $23.5(609)$ & $25.4(382)$ & \\
\hline Education & & & & & & $<0.001$ \\
\hline Junior high or lower & $2.8(899)$ & $3.6(233)$ & $2.6(542)$ & $3.1(79)$ & $3.0(45)$ & \\
\hline High school & $21.6(6,837)$ & $26.9(1,722)$ & $19.2(4,059)$ & $23.6(611)$ & $29.6(445)$ & \\
\hline Vocational school & $15.1(4,780)$ & $13.9(890)$ & $15.0(3,167)$ & $16.9(437)$ & $19.0(286)$ & \\
\hline University or higher & $60.5(19,137)$ & $55.6(3,560)$ & $63.3(13,383)$ & $56.5(1,465)$ & $48.4(729)$ & \\
\hline Country of origin & & & & & & $<0.001$ \\
\hline Taiwan & $94.8(30,002)$ & $91.8(5,877)$ & $95.2(20,133)$ & $97.4(2,524)$ & $97.5(1,468)$ & \\
\hline Other & $5.2(1,651)$ & $8.2(528)$ & $4.8(1,018)$ & $2.6(68)$ & $2.5(37)$ & \\
\hline Employment & & & & & & $<0.001$ \\
\hline Not employed & $38.4(12,166)$ & $45.5(2,914)$ & $36.1(7,645)$ & $37.9(982)$ & $41.5(625)$ & \\
\hline
\end{tabular}

Page 15/19 


\begin{tabular}{|r|r|r|r|l|l|l|} 
Employed & $61.6(19,487)$ & $54.5(3,491)$ & $63.9(13,506)$ & $62.1(1,610)$ & $58.5(880)$ & \\
\hline Newborn sex & & & & & & 0.932 \\
\hline Female & $48.0(15,183)$ & $47.9(3,065)$ & $48.1(10,170)$ & $47.6(1,233)$ & $47.5(715)$ & \\
\hline Male & $52.0(16,470)$ & $52.2(3,340)$ & $51.9(10,981)$ & $52.4(1,359)$ & $52.5(790)$ & \\
\hline Was newborn healthy at birth? & & & & & $<0.001$ \\
\hline Yes & $82.3(26,057)$ & $85.4(5,471)$ & $82.2(17,384)$ & $79.2(2,054)$ & $76.3(1,148)$ & \\
\hline No & $17.7(5,596)$ & $14.6(934)$ & $17.8(3,767)$ & $20.8(538)$ & $23.7(357)$ & \\
\hline
\end{tabular}

\begin{tabular}{|c|c|c|c|c|c|c|c|c|c|c|}
\hline & \multicolumn{3}{|c|}{ Preterm birth } & \multicolumn{2}{|l|}{ SGA } & LGA & \multicolumn{2}{|c|}{ Cesarean section } & \multicolumn{2}{|c|}{ Excessive PWR } \\
\hline & AUC & $\mathrm{p}$-value & AUC & $\mathrm{p}$-value & AUC & $\mathrm{p}$-value & AUC & $\mathrm{p}$-value & AUC & $\mathrm{p}$-value \\
\hline All & & & & & & & & & & \\
\hline Taiwan & 0.64 & $<0.001$ & 0.58 & 0.01 & 0.62 & $<0.001$ & 0.72 & $<0.001$ & 0.68 & $<0.001$ \\
\hline IOM & 0.63 & & 0.59 & & 0.65 & & 0.73 & & 0.69 & \\
\hline Underweight, $\mathrm{BMI}<18.5$ & & & & & & & & & & \\
\hline Taiwan & 0.61 & $<.001$ & 0.59 & 0.597 & 0.64 & 0.502 & 0.53 & 0.081 & 0.66 & 0.693 \\
\hline IOM & 0.57 & & 0.58 & & 0.65 & & 0.54 & & 0.66 & \\
\hline Normal, $18.5<\mathrm{BMI} \leq 24$ & & & & & & & & & & \\
\hline Taiwan & 0.58 & 0.860 & 0.57 & 0.592 & 0.59 & 0.008 & 0.53 & 0.107 & 0.66 & $<0.001$ \\
\hline IOM & 0.58 & & 0.57 & & 0.60 & & 0.54 & & 0.67 & \\
\hline Overweight, $24<\mathrm{BMI} \leq 27$ & & & & & & & & & & \\
\hline Taiwan & 0.56 & 0.924 & 0.55 & 0.901 & 0.56 & 0.855 & 0.55 & 1.000 & 0.69 & 0.026 \\
\hline $\mathrm{IOM}$ & 0.56 & & 0.54 & & 0.55 & & 0.55 & & 0.67 & \\
\hline Obese, $\mathrm{BMI} \geq 27$ & & & & & & & & & & \\
\hline Taiwan & 0.53 & 0.552 & 0.56 & 0.361 & 0.61 & 0.415 & 0.53 & 0.470 & 0.70 & 0.001 \\
\hline IOM & 0.51 & & 0.57 & & 0.60 & & 0.54 & & 0.67 & \\
\hline
\end{tabular}




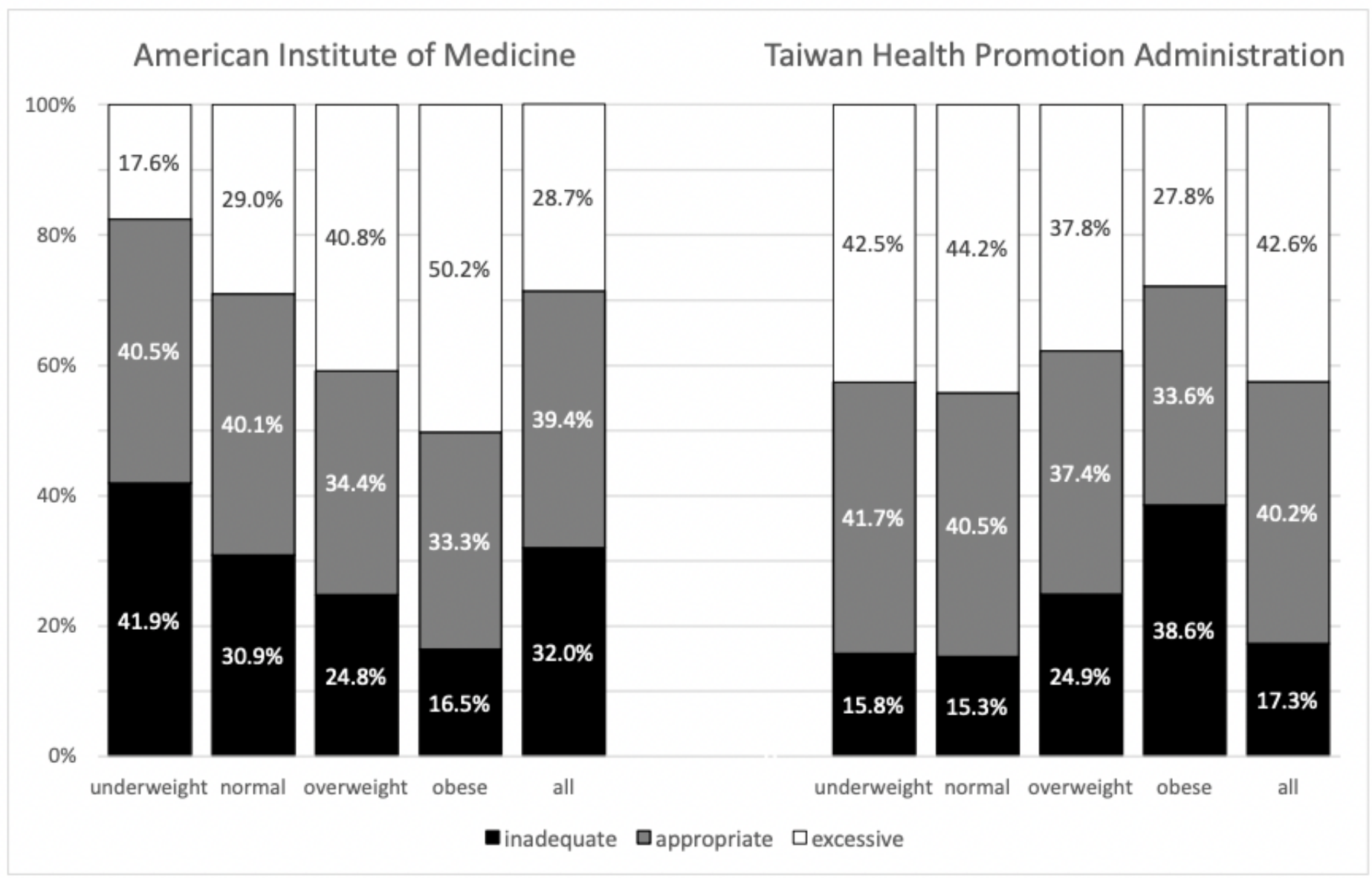

${ }^{\mathrm{a}} 12.5-18 \mathrm{~kg}$ for $\mathrm{BMI}<18.5,11.5-16 \mathrm{~kg}$ for $18.5 \leq \mathrm{BMI}<25,7-11.5 \mathrm{~kg}$ for $25 \leq \mathrm{BMI}<30,5-9 \mathrm{~kg}$ for $\mathrm{BMI} \geq 30$

${ }^{\mathrm{b}} 10-14 \mathrm{~kg}$

${ }^{\mathrm{c}}$ Categorization of body mass index with Taiwan cutoffs of $18.5,24$, and $27 \mathrm{~kg} / \mathrm{m}^{2}$

Figure 2

Distribution of gestational weight gain categories according to the American Institute of Medicinea and the Taiwan Health Promotion Administrationb recommendations among pre-pregnancy body mass index categoriesc $(\mathrm{N}=31653)$. 

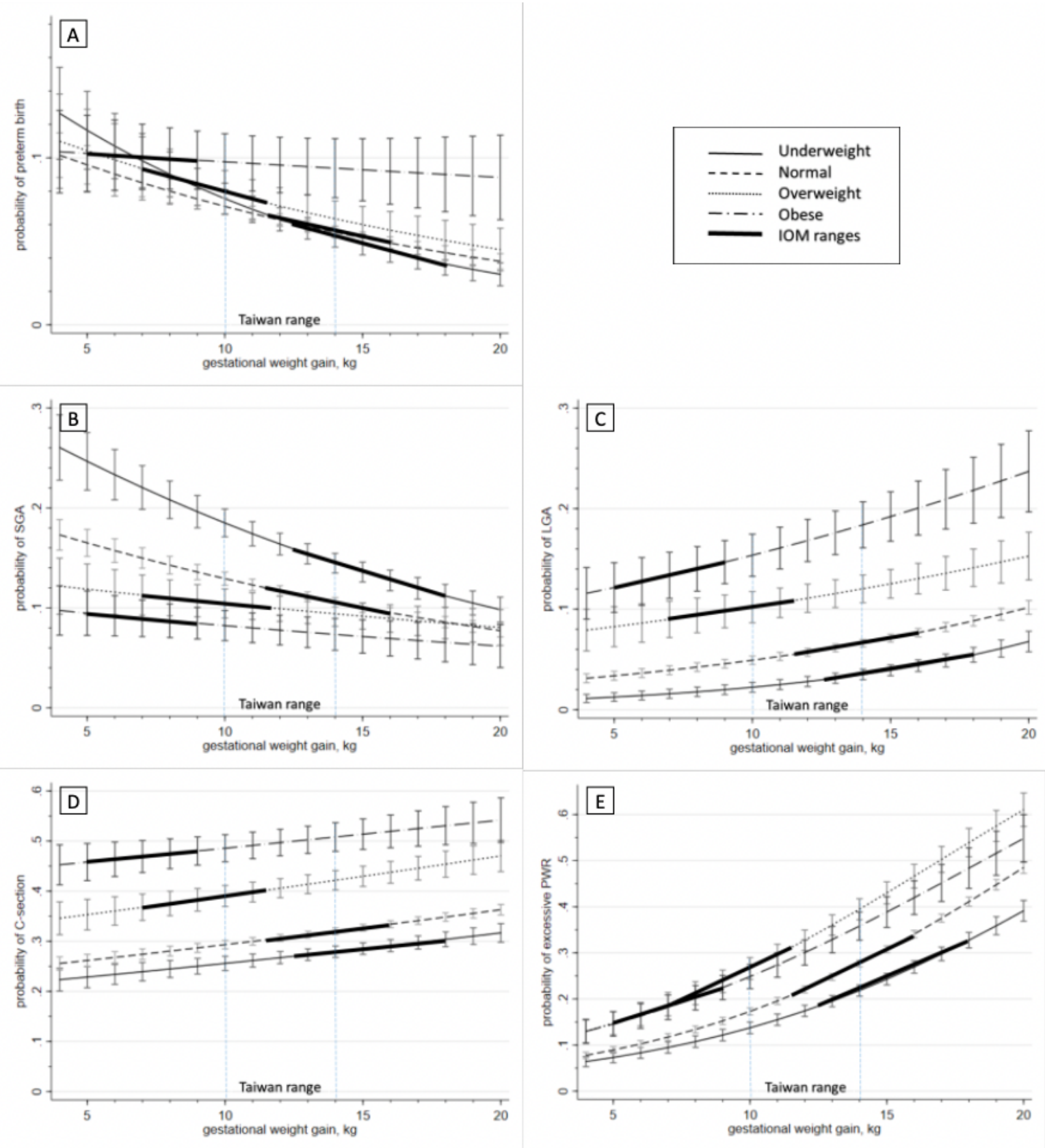

${ }^{*}$ Categorization of body mass index with Taiwan cutoffs of $18.5,24$, and $27 \mathrm{~kg} / \mathrm{m}^{2}$

IOM - American Institute of Medicine, LGA - large for gestational age, SGA - small for gestational age, PWR - postpartum weight retention

\section{Figure 3}

Predicted probabilities and $95 \%$ confidence intervals of adverse birth outcomes based on pre-pregnancy body mass index* $(\mathrm{N}=31653)$.

\section{Supplementary Files}


This is a list of supplementary files associated with this preprint. Click to download.

- Supplementary.docx 